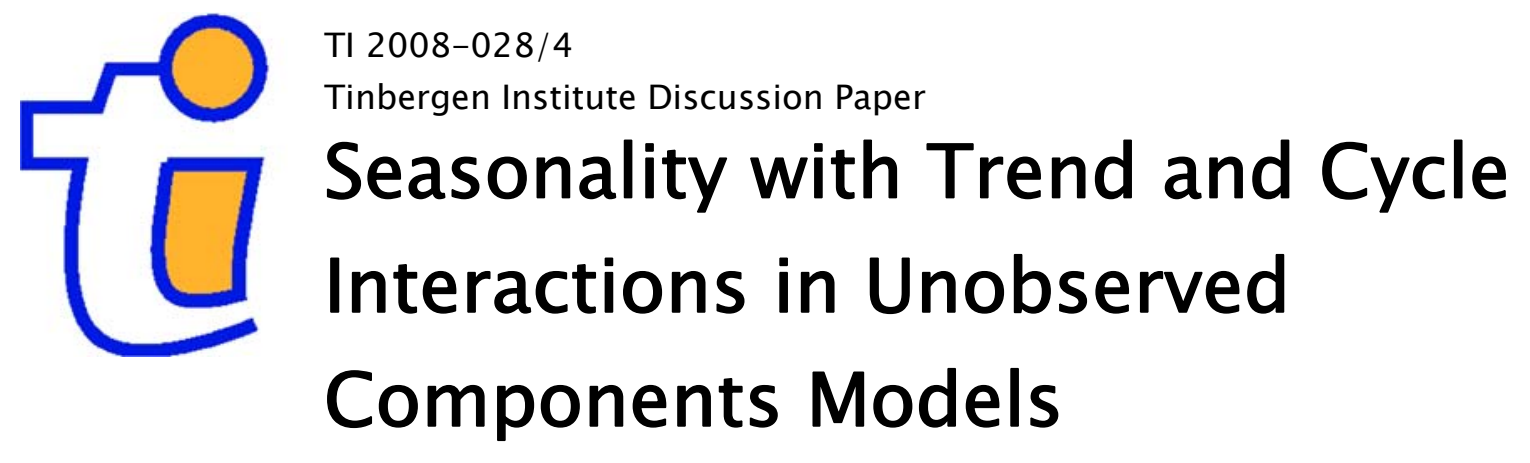

Siem Jan Koopman

Kai Ming Lee 


\section{Tinbergen Institute}

The Tinbergen Institute is the institute for economic research of the Erasmus Universiteit Rotterdam, Universiteit van Amsterdam, and Vrije Universiteit Amsterdam.

Tinbergen Institute Amsterdam

Roetersstraat 31

1018 WB Amsterdam

The Netherlands

Tel.: $\quad+31(0) 205513500$

Fax: $\quad+31(0) 205513555$

Tinbergen Institute Rotterdam

Burg. Oudlaan 50

3062 PA Rotterdam

The Netherlands

Tel.: $\quad+31(0) 104088900$

Fax: $\quad+31(0) 104089031$

Most TI discussion papers can be downloaded at http:/ /www.tinbergen.nl. 


\title{
Seasonality with trend and cycle interactions in unobserved components models
}

\author{
Siem Jan Koopman and Kai Ming Lee \\ VU University Amsterdam, The Netherlands.
}

\begin{abstract}
Summary. Unobserved components time series models decompose a time series into a trend, a season, a cycle, an irregular disturbance, and possibly other components. These models have been successfully applied to many economic time series. The standard assumption of a linear model, often appropriate after a logarithmic transformation of the data, facilitates estimation, testing, forecasting and interpretation. However, in some settings the linear-additive framework may be too restrictive. In this paper, we formulate a non-linear unobserved components time series model which allows interactions between the trend-cycle component and the seasonal component. The resulting model is cast into a non-linear state space form and estimated by the extended Kalman filter, adapted for models with diffuse initial conditions. We apply our model to UK travel data and US unemployment and production series, and show that it can capture increasing seasonal variation and cycle dependent seasonal fluctuations.
\end{abstract}

Keywords: Seasonal interaction; Unobserved components; Non-linear state space models; Extended Kalman filter; Diffuse initialisation.

\section{Introduction}

A common practice in economic time series analyses and seasonal adjustment procedures is first to take logarithms of the data. Linear Gaussian models can often be fitted to the transformed data, while they are inappropriate for the series in the original metric. The log-additive framework appears to work successfully for time series modelling based on the decomposition in trend, seasonal, irregular and other components. The logarithmic transformation converts an exponentialy growing trend into a linear trend. Further it often eliminates or reduces growing seasonal variation and heteroskedasticity in seasonal time series. However, the log-transformation has various drawbacks. In decomposition models or in seasonal adjustment procedures such as the popular X-11 and X-12 programs, the logarithmic transformation presents a single rigid alternative to the untransformed linear-additive specification, see Findley et al. (1998). In particular, it predicates that time series components combine multiplicatively in the implied model for the untransformed series. A full multiplicative model is not always intended or desired. Moreover, when some heteroskedasticity or changing seasonal variation remains after the transformation, applying the log-transformation again is usually not an attractive solution. Finally, if the data is already supplied in units measuring proportional changes, applying the log-transformation can complicate model interpretation.

In cases where the log-transformation does not remove all heteroskedasticity or growth in the seasonal component, an obvious course of action is to test alternative data transformations. In this paper, we explore a different option for the class of unobserved components (UC) models, see Harvey (1989). Searching for an appropriate data transformation is essentially a quest for a suitable functional form of the model. Our approach is to alter the 
functional form directly by relating the seasonal component to other components such as the trend. We introduce a simple multiplicative-additive extension to linear UC models, in which a transformation of the trend acts as a scaling factor to the seasonal component. Estimation is effectively performed using the extended Kalman filter, which is a relatively simple estimation procedure compared to more elaborate simulation-based methods. As our model specification contains non-stationary components, we have adapted an exact diffuse initialisation method to the extended Kalman filter, which is a novelty in the literature. Unlike previous studies with multiplicative seasonality in UC models, we explicitly parameterise and estimate the degree of trend-season interaction. The basic linear form is a simple parameter restriction in our model.

When the data contains a cyclical component, the magnitude of the seasonal influence may vary along the phase of the cycle. Although seasonal fluctuations and business cycles are traditionally assumed to be uncorrelated, for some macro-economic series there is increasing evidence that this assumption is not valid. For example Cecchetti et al. (1997), Franses and de Bruin (1999), van Dijk et al. (2003) and Osborn and Matas-Mir (2004) have found varying amounts of interactions between cycles and seasonal adjustment in unemployment and industrial production series using linear or non-linear smooth transition autoregression models. With a straightforward extension of our trend-season interaction model, we also examine interactions between the seasonal component and the business cycle. Interactions between the season and the trend or the cycle are typically studied separately in the literature. The non-linear UC model allows us to model changes in seasonal variation along both trend and cycle fluctuations, as well as changes resulting from exogenous shocks using a single coherent framework.

In the next section, we describe the basic unobserved components model. We further review models with multiplicative seasonality that have been proposed in the literature. In Secion 3 we introduce our non-linear specification and describe the extended Kalman filter estimation procedure. Empirical applications of the new model are provided in Section 4. We conclude with Section 5 .

\section{The unobserved components time series model}

The unobserved components (UC) time series model has proven to be a valuable tool for seasonal adjustment, see for example Gersch and Kitagawa (1983) and Harvey and Scott (1994). Compared to model-free procedures, they offer the benefit of providing statistical tests and prediction algorithms. Additionally, it is simple to incorporate changing seasonal patterns and to introduce additional features such as explanatory variables, interventions and cyclical components. Estimation of parameters and measurement of the components is based on Kalman filter and smoothing methods which can deal with multivariate series and data irregularities such as missing observations or unevenly recorded data. In this section we briefly introduce the basic form of the model and provide some details which are needed for the following sections.

The seasonal adjustment framework employed in this paper is based on the basic structural model (BSM) as described by Harvey (1989). We assume that the time series $\left\{Y_{t}\right\}$ is observed which we routinely transform into logs, that is

$$
y_{t}=\log Y_{t}, \quad t=1, \ldots, n .
$$


The BSM decomposes $y_{t}$ into additive stochastic components and is given by

$$
y_{t}=\mu_{t}+\gamma_{t}+\varepsilon_{t}, \quad \varepsilon_{t} \sim \operatorname{NID}\left(0, \sigma_{\varepsilon}^{2}\right), \quad t=1, \ldots, n,
$$

where $\mu_{t}$ represents the trend, $\gamma_{t}$ the seasonal component and $\varepsilon_{t}$ the irregular disturbance term. The linear model (2) can be regarded as a generalisation of the classical time series decomposition in which deterministic components for trend and season are replaced by stochastic processes. The BSM is a simple example of an UC model. It can be extended by including deterministic and/or stochastic components. For example, explanatory variables, intervention effects and stochastic cycles can be a part of the UC model.

The trend component $\mu_{t}$ in (2) is specified in our applications by the local linear trend model as given by

$$
\begin{aligned}
\mu_{t+1} & =\mu_{t}+\beta_{t}+\eta_{t}, & & \eta_{t} \sim \operatorname{NID}\left(0, \sigma_{\eta}^{2}\right), \\
\beta_{t+1} & =\beta_{t}+\zeta_{t}, & & \zeta_{t} \sim \operatorname{NID}\left(0, \sigma_{\zeta}^{2}\right),
\end{aligned}
$$

where $\beta_{t}$ represents the drift or slope of the trend $\mu_{t}$ and the disturbances $\varepsilon_{t}, \eta_{t}$ and $\zeta_{t}$ are mutually uncorrelated at all lags and leads, for $t=1, \ldots, n$. Some notable limiting cases of this specification include: if $\sigma_{\zeta} \rightarrow 0$ while $\sigma_{\eta}$ is nonzero the trend is a random walk with drift $\beta_{1}$; if $\sigma_{\eta} \rightarrow 0$ while $\sigma_{\zeta}$ is nonzero the trend follows a smooth integrated random walk; when both tend to zero, $\mu_{t}$ reverts to a deterministic linear trend. In our empirical section we use a smooth trend specification by restricting $\sigma_{\eta}^{2}$ to zero. The initial values of $\mu_{1}, \beta_{1}$ are generally unknown, and will be represented by non-informative or diffuse initial distributions. We will elaborate on this issue in Section 3.4, as the estimation procedure needs to take it into account.

The seasonal component $\gamma_{t}$ can be specified as a sum of time-varying trigonometric cycles. Specifically, in a model for a time series with seasonal length $s$, we have

$$
\left(\begin{array}{c}
\gamma_{j, t+1} \\
\gamma_{j, t+1}^{*}
\end{array}\right)=\left[\begin{array}{rr}
\cos \lambda_{j} & \sin \lambda_{j} \\
-\sin \lambda_{j} & \cos \lambda_{j}
\end{array}\right]\left(\begin{array}{c}
\gamma_{j, t} \\
\gamma_{j, t}^{*}
\end{array}\right)+\left(\begin{array}{c}
\omega_{j, t} \\
\omega_{j, t}^{*}
\end{array}\right), \quad\left(\begin{array}{c}
\omega_{j, t} \\
\omega_{j, t}^{*}
\end{array}\right) \sim \operatorname{NID}\left(0, \sigma_{\omega}^{2} I_{2}\right),
$$

with $\lambda_{j}=2 \pi j / s$ for $j=1, \ldots,[s / 2]$ and $t=1, \ldots, n$. The seasonal disturbances $\omega_{j, t}$ and $\omega_{j, t}^{*}$ are uncorrelated with the previously specified disturbances at all lags and leads. Further details of the seasonal components are discussed by Harvey and Scott (1994) and Proietti (2000) who also describe alternative seasonal component models such as stochastic seasonal dummy variables. Although these alternative specifications can be considered in our non-linear UC model, we restrict ourselves to the trigonometric seasonal component (4) in our study. The seasonal components represent non-stationary processes and their initial conditions rely on diffuse distributions, similar to the trend components.

Many macro-economic time series contain periodic fluctuations of a lower frequency than the seasonal frequencies. For example, fluctuations in economic time series associated with medium frequencies related to periods between 1.5 and 8 years are typically interpreted as the business cycle. The dynamic effects related to these medium frequencies appear often less pronounced in the observed economic time series and tend to be of a stationary nature. To incorporare the cyclical dynamics in the time series model, the BSM can be extended by a stochastic cyclical component $\psi_{t}$. We then have the decomposition model

$$
y_{t}=\mu_{t}+\gamma_{t}+\psi_{t}+\varepsilon_{t}, \quad t=1, \ldots, n,
$$

with

$$
\left(\begin{array}{c}
\psi_{t+1} \\
\psi_{t+1}^{*}
\end{array}\right)=\rho\left[\begin{array}{rr}
\cos \lambda^{c} & \sin \lambda^{c} \\
-\sin \lambda^{c} & \cos \lambda^{c}
\end{array}\right]\left(\begin{array}{c}
\psi_{t} \\
\psi_{t}^{*}
\end{array}\right)+\left(\begin{array}{c}
\kappa_{t} \\
\kappa_{t}^{*}
\end{array}\right), \quad\left(\begin{array}{c}
\kappa_{t} \\
\kappa_{t}^{*}
\end{array}\right) \sim \operatorname{NID}\left(0, \sigma_{\kappa}^{2} I_{2}\right),
$$


where the three unknown coefficients $\lambda^{c}, \rho$ and $\sigma_{\kappa}^{2}$ in the cycle equation (6) represent the cyclical frequency, the damping factor and the cycle disturbance variance, respectively. The period of the cycle is given by $2 \pi / \lambda^{c}$. For $|\rho|<1,0<\lambda<\pi$, the cycle $\psi_{t}$ and the auxilary process $\psi_{t}^{*}$ are stationary $\operatorname{ARMA}(2,1)$ processes, with variance $\sigma_{\kappa}^{2} /\left(1-\rho^{2}\right)$. The cycle collapses into an $\mathrm{AR}(1)$ process when $\lambda^{c}$ approaches zero. The cycle process (6) is stationary when $|\rho|<1$ and its unconditional distribution provides the properly defined initial conditions for $\psi_{t}$ and $\psi_{t}^{*}$. The disturbances $\kappa_{t}$ and $\kappa_{t}^{*}$ are specified to be uncorrelated with the disturbances of the other components at all lags and leads, and uncorrelated with the initial distributions. A more elaborate discussion of the BSM and other UC models is provided by Harvey (1989).

The BSM, possibly extended with a cycle component, can be formulated as a linear state space model specified by the equations

$$
\begin{aligned}
y_{t} & =Z \alpha_{t}+\varepsilon_{t}, \quad \varepsilon_{t} \sim \operatorname{NID}\left(0, \sigma_{\varepsilon}^{2}\right), \\
\alpha_{t+1} & =T \alpha_{t}+\eta_{t}, \quad \eta_{t} \sim \operatorname{NID}(0, H), \quad t=1, \ldots, n,
\end{aligned}
$$

where the first equation relates the observation $y_{t}$ to an unobserved state vector $\alpha_{t}$, which contains the trend, season and other components required for describing the model. The state vector is modelled by the vector autoregressive process specified in the second equation, together with an initial distribution for $\alpha_{1}$. The system variables $Z, T, \sigma_{\varepsilon}^{2}, H$ are chosen to represent a particular model, and will usually depend unknown parameters, which can be estimated by maximising the Gaussian Likelihood function of the model. After replacing the parameters by their estimated values, the unobserved components can be estimated using the Kalman filtering and smoothing equations. The seasonal adjustment procedure based on BSM simply consists of substracting the estimated seasonal component $\gamma_{t}$ from the time series $y_{t}$, that is $y_{t}^{S A}=y_{t}-\hat{\gamma}_{t}$ where $y_{t}^{S A}$ is the seasonally adjusted time series and $\hat{\gamma}_{t}$ is the estimate of $\gamma_{t}$ obtained from the Kalman smoothing equations. The filtering, smoothing and Likelihood equations for linear Gaussian state space models are provided in Appendix A. For a more complete discussion of state space methods and their applications, we refer to Harvey (1989) and Durbin and Koopman (2001). An introductory text for UC models is Commandeur and Koopman (2007).

\section{Seasonal interacting components}

\subsection{A review of non-linear trend-seasonal models}

A mixed additive multiplicative seasonal adjustment procedure based on the classical trendseasonal-irregular decomposition is considered by Durbin and Murphy (1975) for the modeling of a set of unemployment series. The Durbin-Murphy specification is given by

$$
y_{t}=m_{t}+g_{t}+g_{t}^{*} m_{t}+\varepsilon_{t}, \quad \varepsilon_{t} \sim \operatorname{NID}\left(0, \sigma_{\varepsilon}^{2}\right), \quad t=1, \ldots, n,
$$

where $m_{t}$ is a deterministic trend function, $g_{t}$ is an additive seasonal fixed effect and $g_{t}^{*}$ is a multiplicative seasonal fixed factor. A standard moving average filter can be derived to extract the different components from the data. Although this model was not based on a stochastic UC model, it can be regarded as an early precursor to our multiplicative seasonal component model presented in Section 3.2 below.

Bowerman et al. (1990) explored a number of different approaches to deal with the problem of increasing seasonal variation in time series. Although all their suggestions are built on autoregressive moving average model based methods, one of their models takes a 
similar direction to what we propose. In their seasonal interaction model, changes in the seasonal component are directly related to a deterministic trend by

$$
y_{t}=\beta_{0}+\beta_{0}^{+} t+\sum_{j=1}^{s-1} \beta_{j} D_{j, t}+\sum_{j=1}^{s-1} \beta_{j}^{+} D_{j, t} t+u_{t},
$$

where $\beta_{0}+\beta_{0}^{+} t$ is the fixed trend component with unknown coefficients $\beta_{0}$ and $\beta_{0}^{+}, D_{j, t}$ is the seasonal dummy regression variable with unknown coefficients $\beta_{j}$ and $\beta_{j}^{+}$for $j=1, \ldots, s-1$ and $u_{t}$ is modelled as an autoregressive integrated moving average process. The coefficients $\beta_{j}$ are associated with the seasonal effects that are independent of the trend while the coefficients $\beta_{j}^{+}$are interacting with the trend.

In most current applications of UC models, the specifications are of the logarithmic additive type, which can be easily formulated as a linear state space model. An important practical advantage of linearity is that optimal estimates of the latent components, parameters and model predictions are easily obtained using standard Kalman filter based methods. Estimation is a routine procedure for which user-friendly graphical packages are available. Combining multiplicative and additive components may result in a better model fit. However, optimal estimation in such models can be quite complex, and are often carried out using elaborate and computationally expensive simulation methods. For example, Shephard (1994) formulated the multiplicative UC seasonal adjustment model

$$
y_{t}=\left(1+\gamma_{t}+\varepsilon_{t}^{+}\right) \mu_{t}+\varepsilon_{t}, \quad \varepsilon_{t} \sim \operatorname{NID}\left(0, \sigma_{\varepsilon}^{2}\right), \quad \varepsilon_{t}^{+} \sim \operatorname{NID}\left(0, \sigma_{\varepsilon^{+}}^{2}\right),
$$

for $t=1, \ldots, n$, where $\mu_{t}$ and $\gamma_{t}$ are the trend and seasonal components and can possibly be modelled as in (3) and (4), respectively. The two irregular terms $\varepsilon_{t}$ and $\varepsilon_{t}^{+}$are uncorrelated with each other and with all other disturbances at all leads and lags. The seasonal term $\gamma_{t}$ interacts with the trend component through scaling while the irregular term $\varepsilon_{t}^{+}$allows for additional heteroskedasticity. The multiplicative UC model was used to seasonally adjust the UK M4 money supply series based on parameter estimates obtained from Markov chain Monte Carlo methods. Durbin and Koopman (2001) used a similar additive-multiplicative specification as an exposition example for importance sampling techniques.

Proietti and Riani (2006) consider the use of the Box-Cox transformation in seasonal UC models as a generalisation of the log-transformation. This approach implies an inverse Box-Cox transformation on the sum of the components and it allows for a far wider range of options than the usual exponential transformation. However, interpretation in the original metric can be awkward for many values of the Box-Cox transformation parameter. The model was estimated with a combination of numerical integration and simulation techniques.

Finally, the methodology of Ozaki and Thomson (1994) is close to our non-linear UC model of Section 3.2 below although the specifics and motivations of the models are different. Ozaki and Thompson consider a UC model in levels, given by

$$
Y_{t}=M_{t}\left(1+G_{t}\right) e^{\varepsilon_{t}-\sigma^{2} / 2}, \quad \varepsilon_{t} \sim \operatorname{NID}\left(0, \sigma_{\varepsilon}^{2}\right), \quad t=1, \ldots, n,
$$

where $M_{t}$ is a linear Gaussian stochastic process for the trend while $G_{t}$ is a stochastic seasonal component. When the log-transformation is applied to $Y_{t}$, the model for $y_{t}=\log Y_{t}$ becomes linear and is given by

$$
y_{t}=\mu_{t}+\gamma_{t}+\varepsilon_{t}, \quad \text { where } \quad \mu_{t}=\log \left(M_{t}\right)-\sigma^{2} / 2, \quad \gamma_{t}=\log \left(1+G_{t}\right) \text {, }
$$


for $t=1, \ldots, n$. Parameter estimation is carried out on basis of the non-linear model for $Y_{t}$ using the extended Kalman filter rather than fitting the linear model to the log-transformed series $y_{t}$. The main motivation of this approach is to provide a model-based framework for the $\mathrm{X}-11$ seasonal adjustment procedure.

\subsection{Trend and cycle interactions in the basic structural model}

The standard linear BSM of Section 2 is usually fitted to log-transformed data, implying a model with multiplicative components in the untransformed series. In the previous section we have discussed a number of alternative specifications that have been suggested in the literature. These non-linear model specifications can be considered when heteroskedasticity or changing seasonal variation is not adequately removed by the model-based seasonal adjustment procedure. We propose to generalize the BSM by scaling the amplitude of the seasonal component via an exponential transformation of the trend component. The time series, either in levels or in logs, is decomposed by the non-linear model

$$
y_{t}=\mu_{t}+e^{b \mu_{t}} \gamma_{t}+\varepsilon_{t}, \quad \varepsilon_{t} \sim \operatorname{NID}\left(0, \sigma_{\varepsilon}^{2}\right), \quad t=1, \ldots, n,
$$

where $b$ is an unknown fixed coefficient while the dynamic specification of the trend component $\mu_{t}$ is given by (3) and the seasonal component $\gamma_{t}$ is given by (4). The sign of the coefficient $b$ determines whether the seasonal variation increases or decreases when a positive change in the trend occurs. The model reduces to the basic linear specification when $b$ is zero. The overall amplitude of the seasonal component is determined by both $e^{b \mu_{t}}$ and the disturbance variance $\sigma_{\omega}^{2}$ in the stochastic seasonal equation (4). The two sources of seasonal amplitude can be made more explicit by restricting $\sigma_{\omega}^{2}=1$ in (4) and replacing $e^{b \mu_{t}}$ by $e^{a+b \mu_{t}}$ as the scaling process in (8) where $a$ is a fixed unknown coefficient. However, we adopt the specification in (8) to remain close to the original linear BSM.

When the cycle component (6) is added to the BSM we obtain model (5). Similar to the introduction of the trend interaction, we can extend model (5) by a trend and cycle interaction to obtain the non-linear model

$$
y_{t}=\mu_{t}+\psi_{t}+e^{b \mu_{t}+c \psi_{t}} \gamma_{t}+\varepsilon_{t},
$$

where $c$ is an unknown fixed coefficient. The seasonal term in (9) is scaled by an exponential transformation of a linear combination of the trend and cycle components. In economic time series, the $\psi_{t}$ component can often be referred to as the business cycle. In this case, the sign of $c$ determines whether seasonal effects are amplified or dampened during expansions and recessions. The restriction $b=c$ implies that the the seasonal component is scaled by the combined trend-cycle component $\mu_{t}+\psi_{t}$. Model (9) reduces to model (5) when $b=c=0$.

In specification (9) changes to the seasonal pattern can be due to either the random shocks of $\omega_{j, t}$ and $\omega_{j, t}^{*}$ in (4) or to changes in the trend and cycle components. It is possible to generalize the trend-cycle interaction model further. For instance, we can introduce a scaling process to the cyclical component based on the trend and seasonal components. We can also include interactions based on exogenous intervention and regression variables. In this study however we limit ourselves to the specifications described in this section.

\subsection{Seasonal interaction model in state space form}

The non-linear seasonal interaction model cannot be formulated in the linear state space form (7). Therefore we consider a non-linear state space model where the observation 
equation $y_{t}=Z \alpha_{t}+\varepsilon_{t}$ in (7) is replaced by

$$
y_{t}=Z\left(\alpha_{t}\right)+\varepsilon_{t}, \quad \varepsilon_{t} \sim \operatorname{NID}\left(0, \sigma_{\varepsilon}^{2}\right), \quad t=1, \ldots, n .
$$

Here $Z(\cdot)$ is a deterministic non-linear function while the state equation for $\alpha_{t+1}$ in (30) remains. The function $Z(\cdot)$ typically depends on unknown parameters.

The trend and cycle interaction model (9) has a state space representation with a state vector given by

$$
\alpha_{t}=\left(\begin{array}{llllllllllll}
\mu_{t} & \beta_{t} & \psi_{t} & \psi_{t}^{*} & \gamma_{1, t} & \gamma_{1, t}^{*} & \gamma_{2, t} & \gamma_{2, t}^{*} & \cdots & \gamma_{\left[\frac{s-1}{2}\right], t} & \gamma_{\left[\frac{s-1}{2}\right], t}^{*} & \gamma_{[s / 2], t}
\end{array}\right)^{\prime},
$$

for $s$ is even and with the non-linear equation $Z\left(\alpha_{t}\right)$ in (10) given by

$$
Z\left(\alpha_{t}\right)=\mu_{t}+\psi_{t}+e^{b \mu_{t}+c \psi_{t}} \gamma_{t},
$$

for $t=1, \ldots, n$. The dynamic specifications of the components are formulated in the state equation of (30) with system variables given by

$$
T=\left[\begin{array}{cccc}
1 & 1 & 0 & 0 \\
0 & 1 & 0 & 0 \\
0 & 0 & T^{\psi} & 0 \\
0 & 0 & 0 & T^{\gamma}
\end{array}\right], \quad H=\left[\begin{array}{cccc}
0 & 0 & 0 & 0 \\
0 & \sigma_{\zeta}^{2} & 0 & 0 \\
0 & 0 & \sigma_{\kappa}^{2} I_{2} & 0 \\
0 & 0 & 0 & \sigma_{\omega}^{2} I_{s-1}
\end{array}\right],
$$

where

$$
T^{\psi}=\rho C\left(\lambda^{c}\right), \quad T^{\gamma}=\operatorname{diag}\left\{C\left(\lambda_{1}\right) \quad \ldots \quad C\left(\lambda_{\left[\frac{s-1}{2}\right]}\right) \quad-1\right\},
$$

for $s$ is even and with $\lambda_{j}=\frac{2 \pi j}{s}$ for $j=1, \ldots,[s / 2]$ and

$$
C(\lambda)=\left[\begin{array}{cc}
\cos \lambda & \sin \lambda \\
-\sin \lambda & \cos \lambda
\end{array}\right]
$$

The elements of the initial state vector are diffuse except for $\psi_{t}$ and $\psi_{t}^{*}$ which represent stationary variables. We therefore have $\alpha_{1} \sim \mathrm{N}\left(a_{1}, P_{1}\right)$ with

$$
a_{1}=0, \quad P_{1}=\left[\begin{array}{cccc}
k & 0 & 0 & 0 \\
0 & k & 0 & 0 \\
0 & 0 & \frac{1}{1-\rho^{2}} \sigma_{\kappa}^{2} I_{2} & 0 \\
0 & 0 & 0 & k I_{s-1}
\end{array}\right]
$$

and let $k \rightarrow \infty$. The state space formulation of the seasonal component with an odd seasonal length is discussed in Durbin and Koopman (2001).

\subsection{Parameter estimation by the extended Kalman filter}

Estimation of the parameters and unobserved components in the BSM usually proceeds by the procedure outlined at the end of Section 2. However, for the non-linear seasonal interaction model (9) the Kalman filter cannot be applied directly. Many methods to estimate non-linear state space models have been proposed in various disciplines. Early algorithms were based on linearization of the non-linear functions. Most of the recent work concentrate on simulation-based methods such as importance sampling and Markov chain 
Monte Carlo methods, see Fruhwirth-Schnatter (1994), Carter and Kohn (1994), Shephard and Pitt (1997) and Durbin and Koopman (2000). In the engineering disciplines, the simulation-based particle filter is often employed for analyzing non-linear state space models, see Gordon et al. (1993), Pitt and Shephard (1999) and de Freitas et al. (2001). In this paper we use the extended Kalman filter (EKF) for state estimation and likelihood evaluation. The EKF has the virtue of being relatively simple in concept and implementation while modest in terms of computational requirements. A review of the EKF method is provided in Anderson and Moore (1979).

The Kalman filter evaluates the conditional expectation of the state vector $\alpha_{t}$ given past observations $y_{1}, \ldots, y_{t-1}$ or given past and concurrent observations $y_{1}, \ldots, y_{t}$. The evaluation of these state estimates is an intractable problem for general non-linear state space models. However, in many specific cases a practical approximation can be found. The extended Kalman filter is based on a linearization of the non-linear effects in the model. For the seasonal interaction model, the smooth non-linear function $Z(\cdot)$ is expanded around an estimate of the state vector which we obtain from the Kalman filter. Since the Taylor approximation is linear in the state vector $\alpha_{t}$, it can be estimated using the standard linear Kalman filter. The Kalman filter applied to the linearized model is termed the extended Kalman filter (EKF) for the non-linear state space model. The appendix provides details of the EKF and its incorporation into the Kalman filter.

The EKF generally provides suboptimal estimates of the state vector in the original non-linear model. In the engineering disciplines, from where the EKF originates, filtering is regularly used to track physical objects. The researcher is typically more certain about the model since it is derived from physical principles. The use of more sophisticated nonlinear filtering techniques is therefore helpful to obtain more precise estimates. In economic applications however, models are seldomly interpreted as true descriptions of the underlying dynamics. The non-linear model is adopted to develop methods for improving the model fit compared to the linear specification. This inaccuracy due to the linearization step in the EKF does not need to be interpreted as an error. Nevertheless, when more accurate state estimates for the non-linear specification are desired, we can employ more computationally demanding simulation methods.

An important issue in estimating stochastic trends and seasonal components is the treatment of the initial state vector $\alpha_{1}$ with mean $a_{1}$ and variance $P_{1}$ which are given by (16) for the seasonal interaction model. The trend and seasonal components are non-stationary processes and we therefore treat the associating elements in the state vector as diffuse variables, that is $k \rightarrow \infty$ in (16). In practice, a simple approach is to replace $k$ by a very large numerical value in (16), say $k=10^{7}$. In our calculations, this approach has been detrimental to the numerical stability of the estimation procedure. We therefore have adopted the exact diffuse initialisation algorithms of Koopman and Durbin (2003) and we have modified the EKF accordingly. Details of the diffuse recursions for the EKF together with an expression for the diffuse likelihood and the smoothing equations for the seasonal interaction model are given in the Appendix B.

The maximum likelihood estimates in this paper are obtained by maximising of the likelihood function using the numerical maximisation routines of the Ox matrix programming language by Doornik (2007). The diffuse EKF routines were programmed in Ox, with support from the functions in the suite of SsfPack routines by Koopman et al. (1999). 

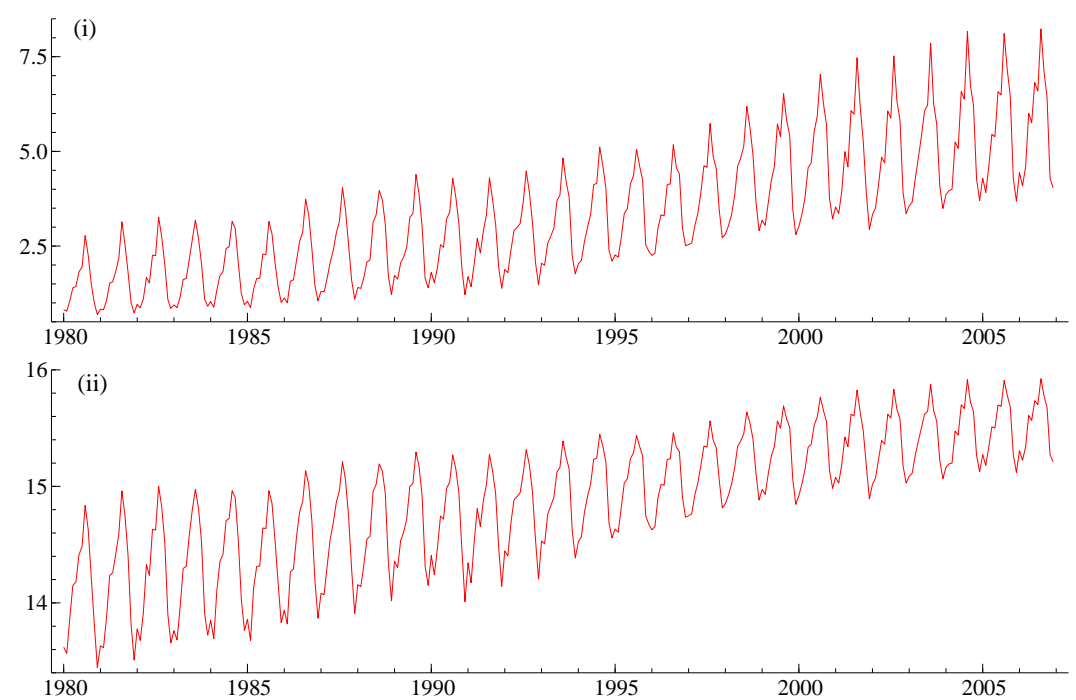

Fig. 1. Visist of UK residents abroad (i) in levels (million); (ii) in logarithms.

\section{Applications}

\subsection{UK visits abroad}

We consider a dataset of monthly visits abroad by UK residents from January 1980 to December 2006. The data is compiled by the Office for National Statistics (ONS), based on the International Passenger Survey. Figure 1 shows plots of the series in levels and logs. The time series of visits abroad shows a clear upwards trend, a pronounced seasonal pattern, and a steady increase of the seasonal variation over time. However, after applying the logtransformation, the increase of seasonal variation has been converted into a decrease. This may indicate that the log transformation is not particularly appropriate for this series.

A linear UC model with smooth trend, trigonometric seasonal and cycle components together with a Normal white noise disturbance term as given by equations (3), (4), (5) and (6) is considered first for the number of visitors in levels. The maximum likelihood estimates of the parameters are

$$
\begin{aligned}
& \hat{\sigma}_{\varepsilon}=0.106, \quad \hat{\sigma}_{\zeta}=0.00062, \quad \hat{\sigma}_{\omega}=0.0119, \\
& \hat{\sigma}_{\kappa}=0.00050, \quad \hat{\rho}=0.958, \quad 2 \pi / \hat{\lambda}^{c}=123, \quad \log L=48.2,
\end{aligned}
$$

where $\log L$ is the $\log$-likelihood value of the model evaluated at the maximum likelihood estimates of the parameters. The estimated standard deviation of the trend disturbance is relatively small, which implies that the trend is quite steady. The cycle has the period $2 \pi / \lambda^{c}$ which is estimated by 123 months. Furthermore, the estimates of the cycle parameters include a relatively small disturbance and amplitude. Most of the variation in the series can be attributed to the seasonal component and to the disturbance term.

Some diagnostic tests based on the standardized one-step ahead prediction errors $f_{t}^{-1 / 2} v_{t}$ 
are

$$
N\left(\chi_{2}^{2}\right)=6.21, \quad H_{104}\left(F_{104,104}\right)=2.46, \quad Q_{12}\left(\chi_{11}^{2}\right)=30.9, \quad Q_{24}\left(\chi_{23}^{2}\right)=43.8,
$$

where $N$ is a Normality statistic based on the third and fourth moments, $H_{m}$ a heteroskedasticity statistic based on the ratio of sample variances for the first and last one third of the prediction errors, and $Q_{l}$ the Box-Ljung serial correlation statistic up to $l$ lags. The null distributions of the tests are given between parentheses. The diagnostics indicate that there is significant residual heteroskedasticity and serial correlation in the estimated model.

Next the non-linear specification

$$
y_{t}=\mu_{t}+\psi_{t}+e^{b \mu_{t}} \gamma_{t}+\varepsilon_{t}
$$

is considered, for which parameter estimates are obtained by by applying the EKF from Section 3.2. The parameter and likelihood estimates are given by

$$
\begin{gathered}
\hat{\sigma}_{\varepsilon}=0.116, \quad \hat{\sigma}_{\zeta}=0.00090, \quad \hat{\sigma}_{\omega}=0.00611, \quad \hat{b}=0.0984 \\
\hat{\sigma}_{\kappa}=0.00088, \quad \hat{\rho}=0.921, \quad 2 \pi / \hat{\lambda}^{c}=589, \quad \log L=55.1 .
\end{gathered}
$$

The most striking difference between the estimates of the linear model and model (19) is the large drop in the value of the standard deviation of the seasonal disturbances, from 0.0119 to 0.00611 . The seasonal component $\gamma_{t}$ in the non-linear model is scaled by the process $e^{b \mu_{t}}$, which must account for most of the drop in $\sigma_{\omega}$. The variation in $\mu_{t}$ is now factored in the seasonality through the scaling in $e^{b \mu_{t}} \gamma_{t}$. The process for $\gamma_{t}$ itself fluctuates less as a result. The upper graph of figure 2 illustrates this by showing the scaled $\left(e^{b \mu_{t}} \gamma_{t}\right)$ and unscaled $\left(\gamma_{t}\right)$ seasonal components as estimated by the extended Kalman smoother. The scaled component is changing largely due to the trend component, while the unscaled component shows much smaller movements and consequently does not require a large standard deviation in the disturbance. We confirm that the scaled component is roughly the same as the estimated $\gamma_{t}$ from the linear model. In the non-linear model, the cycle frequency $\lambda^{c}$ approaches zero, which implies that the period approaches infinity and the the cycle process $\psi_{t}$ reduces to a first order autoregressive process.

The diagnostic tests for the residuals of the non-linear model are given by

$$
N\left(\chi_{2}^{2}\right)=3.18, \quad H_{104}\left(F_{104,104}\right)=1.85, \quad Q_{12}\left(\chi_{11}^{2}\right)=21.9, \quad Q_{24}\left(\chi_{23}^{2}\right)=31.0 .
$$

Compared to the previous linear model, all the diagnostic tests have improved. The $Q_{12}$ and the $H$ statistic are still significant at the $5 \%$ level, but autocorrelation and heteroskedasticity are less severe than they were in the initial specification. Taken together with the significant increase in the log-likelihood, we conclude that the non-linear model is a clear improvement over the linear specification.

\subsection{US unemployment}

In this section we apply the seasonal interaction model to the log of the number of unemployed persons in the US. The monthly data set was obtained from the Bureau of Labor Statistics and spans the period from Januari 1948 to December 2006.

A graph of the log-unemployment with the estimated trend from a linear decomposition model with trend, season, cycle and irreglar components is shown in Figure 3. Salient 


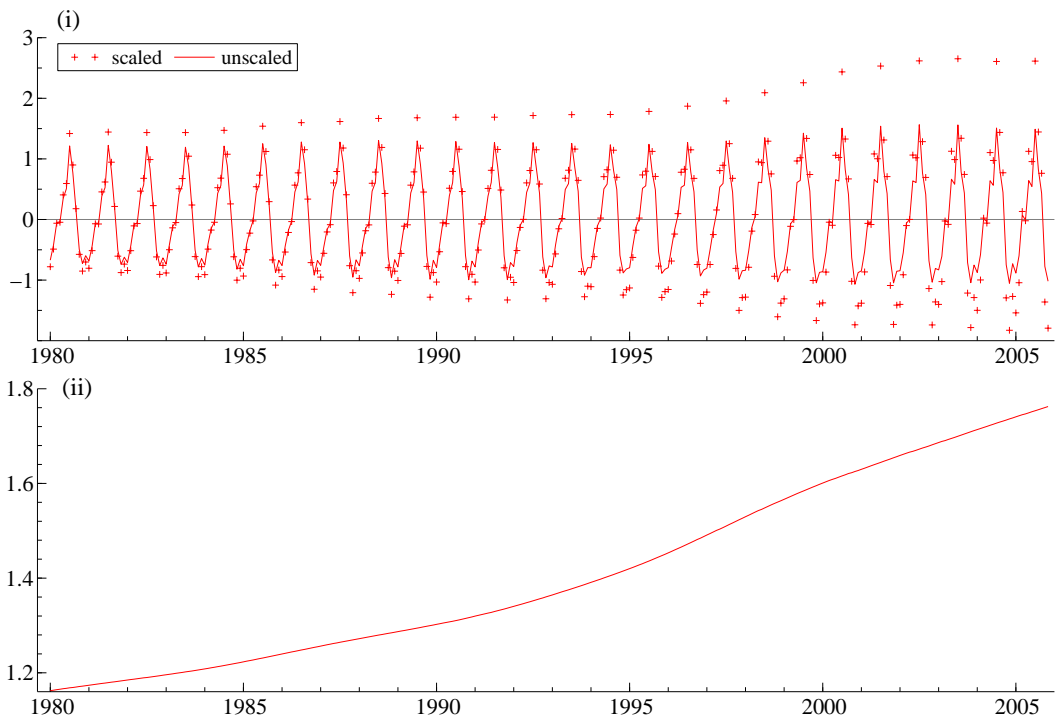

Fig. 2. Visits of UK residents abroad: (i) smooth estimates of the scaled and unscaled seasonal components obtained by the EKF and its associated smoothing equations; (ii) scaling process $e^{b \mu_{t}}$ with $\mu_{t}$ replaced by its smoothed estimate.

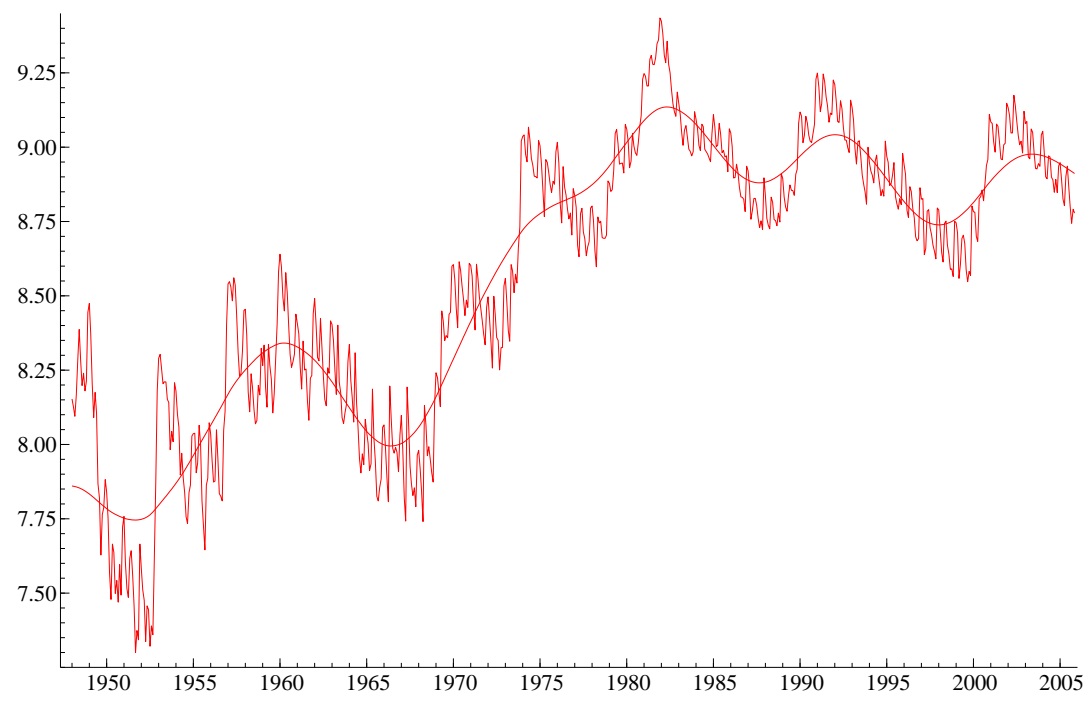

Fig. 3. US unemployed persons with smooth trend obtained by the Kalman smoother, applied to a linear trend-cycle-season-irregular UC decomposition model. 
features in the series are an overall increasing trend that levels off towards the end, a distinct seasonal pattern, and a large amount of medium frequency cyclical fluctuation. The estimated parameters from the linear decomposition model considered are given by

$$
\begin{array}{r}
\hat{\sigma}_{\varepsilon}=0.00034, \quad \hat{\sigma}_{\zeta}=0.00150, \quad \hat{\sigma}_{\omega}=0.00120, \\
\hat{\sigma}_{\kappa}=0.00072, \quad \hat{\rho}=0.970, \quad 2 \pi / \hat{\lambda^{c}}=57, \quad \log L=1082.3,
\end{array}
$$

with diagnostics

$$
N\left(\chi_{2}^{2}\right)=70.6, \quad H_{228}\left(F_{228,228}\right)=4.43, \quad Q_{12}\left(\chi_{11}^{2}\right)=18.5, \quad Q_{24}\left(\chi_{23}^{2}\right)=33.9 .
$$

The business cycle is quite persistent, with a damping factor of 0.97 for a monthly frequency, which corresponds to 0.7 for a yearly frequency. The period of the cycle is close to five years, which is a typical business cycle frequency. In Figure 3 The estimated trend is displayed, and it may be concluded that the series possibly contains a second cycle with a longer period that is currently captured by the trend component. The prediction error based diagnostic tests indicate that Normality and homoskedasticity are strongly rejected, while the serial correlation statistics are not significant at the $5 \%$ level.

The non-linear model with interactions between the trend plus cycle and the seasonal component is given by equation (9) and is considered next. First we concentrate on the cycle-season interaction and estimated the parameters of this model under the constraint $b=0$. Maximum likelihood estimates are given by

$$
\begin{gathered}
\hat{\sigma}_{\varepsilon}=0.00034, \quad \hat{\sigma}_{\zeta}=0.00217, \quad \hat{c}=-0.58 \quad \hat{\sigma}_{\omega}=0.00117, \\
\hat{\sigma}_{\kappa}=0.00065, \quad \hat{\rho}=0.968, \quad 2 \pi / \hat{\lambda^{c}}=53, \quad \log L=1098.0,
\end{gathered}
$$

with diagnostics

$$
N\left(\chi_{2}^{2}\right)=47.3, \quad H_{228}\left(F_{228,228}\right)=4.22, \quad Q_{12}\left(\chi_{11}^{2}\right)=15.0, \quad Q_{24}\left(\chi_{23}^{2}\right)=32.5 .
$$

Compared to the estimates from the linear model, the cycle lenght becomes slightly shorter. The model fit has improved in terms of the increase in the likelihood function. The diagnostic tests show small improvements, but the Normality and heteroskedasticity tests remain highly significant. The negative value of the estimated coefficient $\hat{c}$ indicates that the seasonal component is dampened during periods of high cyclical unemployment and attenuated in the negative phases of the cycle, which is consistent with the findings of Franses (1995). The smoothed scaling process is depicted in Figure 4, together with the scaled and unscaled seasonal component. The plot shows that the scaling process adds about $20 \%$ cyclical variation to $\gamma_{t}$ in the early parts of the series, but levels off towards the end as the amplitude of the estimated cycle component wanes in the last decades.

We estimate a non-linear model with both trend-season and cycle-season interactions next by relaxing the restriction $b=0$. The parameters estimates are given by

$$
\begin{array}{r}
\hat{\sigma}_{\varepsilon}=0.00033, \quad \hat{\sigma}_{\zeta}=0.00050, \quad \hat{b}=-0.024, \quad \hat{c}=-0.53 \quad \hat{\sigma}_{\omega}=0.00126, \\
\hat{\sigma}_{\kappa}=0.00074, \quad \hat{\rho}=0.980, \quad 2 \pi / \hat{\lambda}^{c}=76, \quad \log L=1106.9,
\end{array}
$$

with diagnostics

$$
N\left(\chi_{2}^{2}\right)=49.2, \quad H_{228}\left(F_{228,228}\right)=4.47, \quad Q_{12}\left(\chi_{11}^{2}\right)=23.3, \quad Q_{24}\left(\chi_{23}^{2}\right)=45.9 .
$$



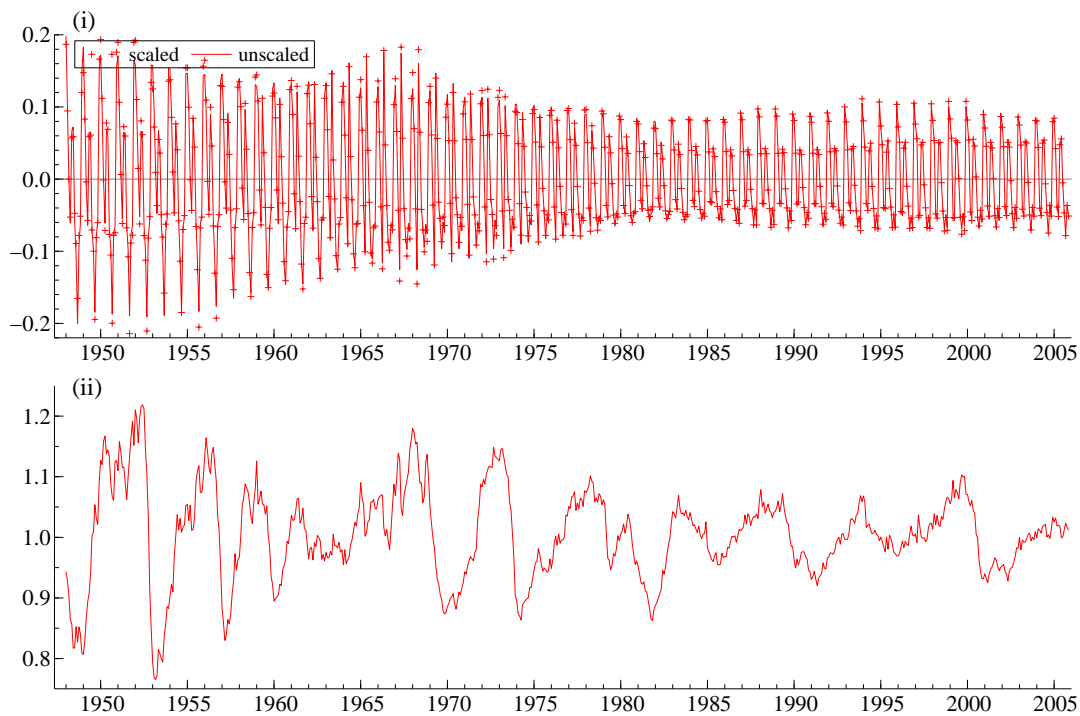

Fig. 4. US unemployed persons: (i) scaled and unscaled seasonal component; (ii) scaling process.

Although there is a considerable increase in the likelihood, not all the diagnostic statistics have improved. The most notable difference is that the serial correlation in the residuals is more severe than it is in the original linear specification, and is now significant at the $5 \%$ level. This may be attributed to the fact that compared to previous estimates, there is a considerable change in the decomposition, as the trend is smoother, while the cycle period has lengthened over six years. Thus, a direct comparison with the linear specification is difficult, in contrast to the previous non-linear specification without a trend-season interaction. If we fix the cycle length at the value of the previous model and re-estimate, we obtain the estimates

$$
\begin{array}{r}
\hat{\sigma}_{\varepsilon}=0.00034, \quad \hat{\sigma}_{\zeta}=0.00143, \quad \hat{b}=-0.021, \quad \hat{c}=-0.60 \quad \hat{\sigma}_{\omega}=0.00132, \\
\hat{\sigma}_{\kappa}=0.00067, \quad \hat{\rho}=0.973, \quad 2 \pi / \lambda^{c}=53, \quad \log L=1104.6,
\end{array}
$$

with diagnostics

$$
N\left(\chi_{2}^{2}\right)=49.4, \quad H_{228}\left(F_{228,228}\right)=4.04, \quad Q_{12}\left(\chi_{11}^{2}\right)=17.6, \quad Q_{24}\left(\chi_{23}^{2}\right)=34.2 .
$$

These diagnostic test statistics are very close to those from the model with only cycleseason interactions, while the likelihood still shows a significant improved. Thus, we prefer this decomposition to the previous one with an unrestricted cycle frequency parameter. Nevertheless, the diagnostics are still not quite satisfactory for this series. As noted by Franses (1995), the US unemployment series exhibits several types of non-linearities that we have not incorporated in our model, such as asymmetry in the cycle and different shock persistence in different regimes. A complete treatment will likely require a more elaborate model, which we consider beyond the scope of this paper. 
Table 1. US monthly industrial and dwellings production, linear and non-linear model estimates and test statistics.

\begin{tabular}{|lrrrrrrr|}
\hline$\left(\hat{\sigma} \times 10^{-3}\right)$ & \multicolumn{1}{c}{ industrial production } & & \multicolumn{5}{c|}{ dwellings production } \\
\cline { 2 - 5 } \cline { 6 - 7 } & linear & trend-cycle & linear & trend & cycle & trend-cycle \\
\hline$\hat{\sigma}_{\varepsilon}$ & 0.0007 & 0.0007 & 0.001 & 0.001 & 0.001 & 0.001 \\
$\hat{\sigma}_{\zeta}$ & 0.433 & 0.450 & 0.388 & 0.408 & 0.561 & 0.466 \\
$\hat{\sigma}_{\omega}$ & 0.223 & 0.223 & 0.574 & 0.680 & 0.555 & 0.640 \\
$\hat{\sigma}_{\kappa}$ & 0.029 & 0.029 & 0.236 & 0.232 & 0.232 & 0.230 \\
$2 \pi / \hat{\lambda}^{c}$ & 60 & 60 & 73 & 72 & 72 & 72 \\
$\hat{\rho}$ & 0.97 & 0.97 & 0.99 & 0.99 & 0.99 & 0.99 \\
$\hat{b}$ & - & 0.0009 & - & -0.063 & - & -0.052 \\
$\hat{c}$ & - & -0.063 & - & - & -0.289 & -0.245 \\
$\log L$ & 1742.0 & 1742.6 & 1182.7 & 1186.0 & 1187.5 & 1189.8 \\
$N$ & 15.5 & 16.5 & 14.9 & 12.2 & 16.8 & 12.6 \\
$H$ & 2.18 & 2.16 & 2.49 & 2.48 & 2.40 & 2.41 \\
$Q_{24}$ & 34.7 & 35.2 & 130.7 & 128.1 & 120.6 & 120.5 \\
$Q_{48}$ & 76.7 & 77.7 & 146.3 & 142.6 & 142.3 & 140.7 \\
\hline
\end{tabular}

\subsection{US industrial and dwellings production}

In our final empirical application we consider the our seasonal interaction model for the US industry and dwellings production series, obtained from OECD Main Economic Indicators 2007 release 07. Both monthly series start in Januari 1960 and end in December 2006. The production of total industry is an index series standardised at 100 in the year 2000, while the production of dwellings is measured in billion US dollars. We model both series in logarithms.

Table 1 presents the estimation results of linear and non-linear models for both series. For the industrial production series, we allow both trend-season and cycle-season interactions in the non-linear model. The estimates show that there is almost no improvement resulting from using the more general non-linear specification. We therefore conclude that no trend or cycle induced variations in the seasonal component of the US industrial production series is detected by our model.

For the dwelling production series, we estimate the parameters for non-linear models with only a trend-season interaction $(c=0)$, only a cycle-season interaction $(b=0)$ and with both trend-season and cycle-season interactions. We learn from Table 1 that both forms of seasonal interactions improve upon the linear model, and either is significant on its own as judged by a Likelihood Ratio test. The estimated coefficient of the trendseason interaction is negative, which implies that the seasonal variation decreases with an increase in the trend. It can be argued that technological changes which may have reduced seasonal dependence in dwellings productions in the past decades have coincided with the trending behaviour in the series, which are likely caused by many unrelated factors such as demographic trends or changing preferences. Our model does not distinguish between underlying causes, but merely reflects the effect of permitting the interaction.

The negative coefficient of the cycle-season interaction indicates that the seasonality in the dwellings productions moves contra-cyclical, that is, the seasonal amplitude decreases with a upswings in the production. A similar effect has been documented in some other US industries by Cecchetti et al. (1997), who interpret it as a capacity constraint in the sector when the inventory series of the sector does not show a decrease. However, in this paper we do not model inventory series . 

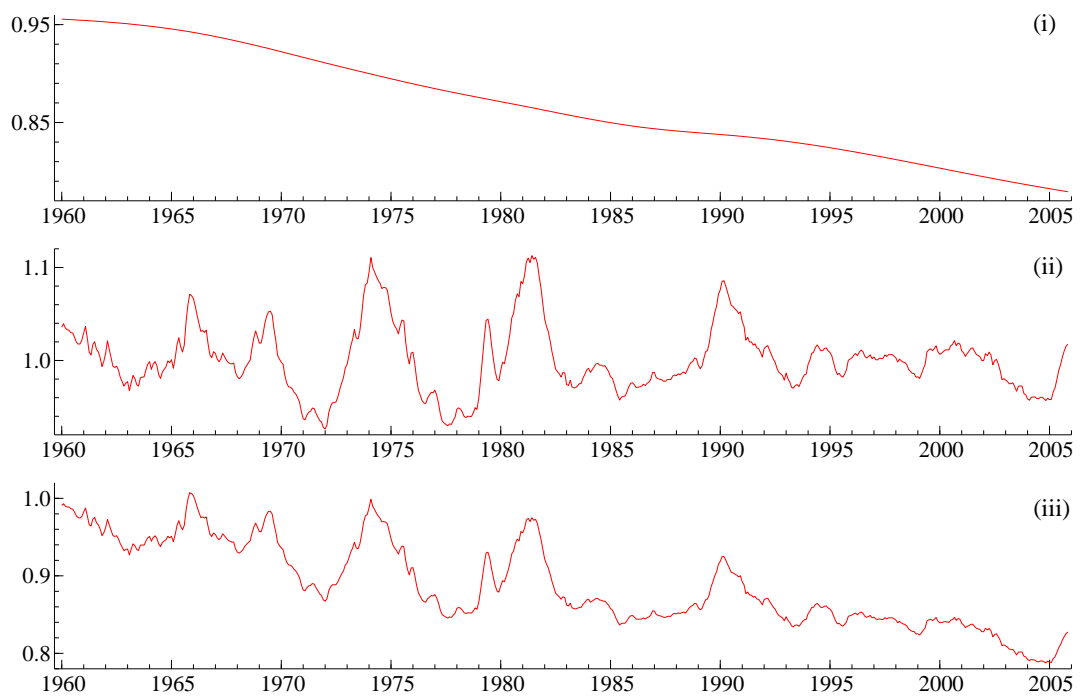

Fig. 5. US dwellings production, seasonal scaling proces from non-linear model with (i) trend interaction; (ii) cycle interaction; (iii) trend-cycle interaction.

Figure 5 shows the estimated scaling process $\exp \left(b \mu_{t}+c \psi_{t}\right)$ from all three non-linear models. We observe that the trend induced reduction in the seasonality is fairly uniform and is roughly $20 \%$ over the sample period. The cyclical swings contribute about $10 \%$ at their top in the mid 1970s and early 1980s. The cycle induced variations in seasonality seems to have reduced since the early 1990s, as a direct result of the declining amplitude of the cyclical component. Finally, we present the unobserved components decomposition of the non-linear model with both trend-season and cycle-season interactions in figure 6 . The figure includes the data and estimated trend, cycle scaled and unscaled seasonal component.

\section{Conclusion}

In this paper we have presented a simple non-linear extension to the basic unobserved components model to allow the seasonal term to interact with the trend and cycle components. The model that we propose addresses some functional misspecifications that may arise from imposing a (transformed) additive components structure. Starting from a basic linear unobserved components model with trend, season, cycle and irregular components, we include a transformed linear combination of trend and cycle components as a scaling factor for the seasonal component. In the resulting model, the seasonal amplitude is amplified or dampened along movements of the trend and cycle, depending on the estimated parameter.

In our empirical applications, we have considered models for UK travel, US unemployment and US production data. The travel data contains increasing seasonal variation, which is not adequately removed by a logarithmic transformation. Our non-linear model shows a significant improvement in the model fit and provides better residual diagnostics. In the unemployment series, we found significant interactions between the cycle and the seasonal term. Although the model improves on the linear specification, it does not capture all the 

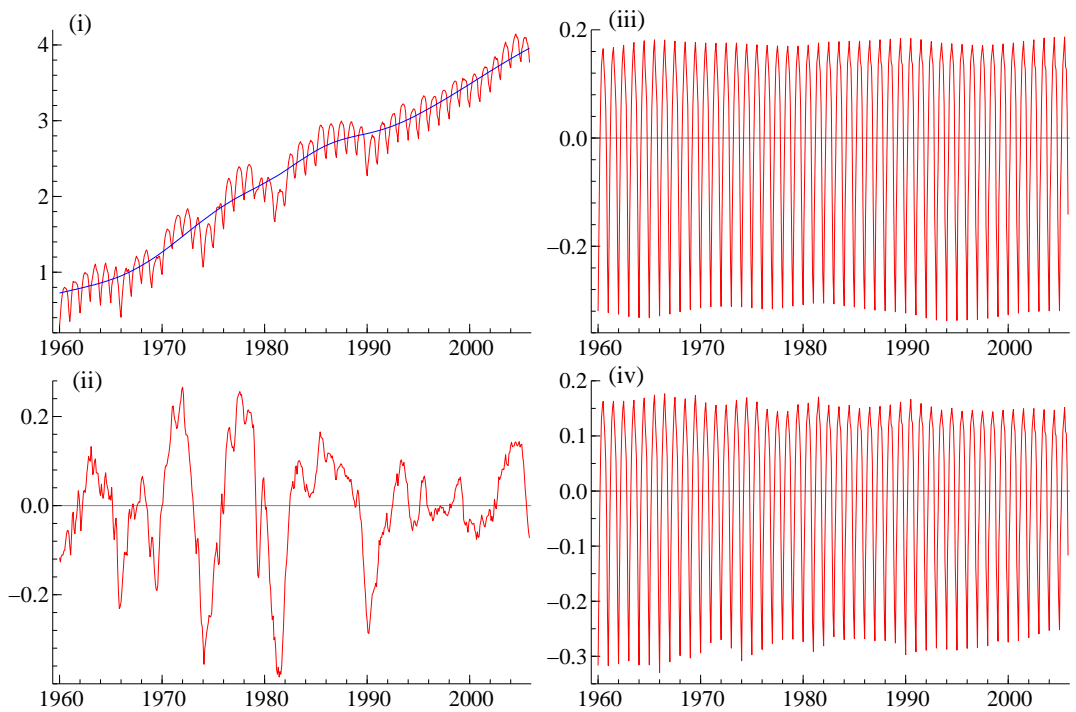

Fig. 6. Smooth estimates of non-linear UC decomposition of US dwellings production with interaction between the seasonal and the trend and cycle components: (i) data and trend; (ii) cycle; (iii) unscaled season (iv) scaled season.

non-linear dynamics in the series. The estimated coefficient sign indicates that seasonal effects are dampened during recessions. Finally our parameter estimates for the US production series do not show evidence of interactions between in the total productions series. However, in the production of dwellings, we observe a significant contra-cyclical effect, as well as a dampening of seasonal fluctuations along the increasing trend. 


\section{A. Kalman filter methods}

A general formulation of a univariate linear Gaussian state space model is given by

$$
\begin{aligned}
& y_{t}=c_{t}+Z_{t} \alpha_{t}+\varepsilon_{t}, \quad \varepsilon_{t} \sim \operatorname{NID}\left(0, \sigma_{\varepsilon}^{2}\right), \\
& \alpha_{t+1}=d_{t}+T_{t} \alpha_{t}+\eta_{t}, \quad \eta_{t} \sim \operatorname{NID}\left(0, H_{t}\right), \quad t=1, \ldots, n,
\end{aligned}
$$

where the first equation relates the scalar observation $y_{t}$ to the state vector $\alpha_{t}$ which is modelled as the VAR(1) process as given in the second equation. The state vector contains the unobserved components and additional variables to enable the specification of the dynamic processes of the components. The disturbance scalar $\varepsilon_{t}$ and the disturbance vector $\eta_{t}$ are assumed to be uncorrelated at all times. The scalars $c_{t}$ and $\sigma_{\varepsilon}^{2}$, the vectors $Z_{t}$ and $d_{t}$ and the matrices $T_{t}$ and $H_{t}$ are fixed system variables which are designed to represent a particular model. Some of the system variables may depend on a unknown parameters which we collect in the vector $\theta$. The initial state vector is distributed as $\alpha_{1} \sim \mathrm{N}\left(a_{1}, P_{1}\right)$.

The well-known Kalman filter equations for the state space model (30) are given by

$$
\begin{aligned}
v_{t} & =y_{t}-Z_{t} a_{t}-c_{t}, & f_{t} & =Z_{t} P_{t} Z_{t}^{\prime}+\sigma_{\varepsilon}^{2}, \quad K_{t}=P_{t} Z_{t}^{\prime} f_{t}^{-1}, \\
a_{t \mid t} & =a_{t}+K_{t} v_{t}, & P_{t \mid t} & =P_{t}-f_{t} K_{t} K_{t}^{\prime}, \\
a_{t+1} & =d_{t}+T_{t} a_{t \mid t}, & P_{t+1} & =T_{t} P_{t \mid t} T_{t}^{\prime}+H_{t},
\end{aligned}
$$

for $t=1, \ldots, n$, where $v_{t}$ is the one-step ahead prediction error with mean square error (MSE) $f_{t}, K_{t}$ is the Kalman gain vector and $a_{t \mid t}$ and $a_{t+1}$ are conditional expectations of the state vector $\alpha_{t}$ with MSE matrices $P_{t \mid t}$ and $P_{t+1}$, respectively. The Kalman filter recursions provide an efficient method for computing the filtered state $a_{t \mid t}$ and the predicted state $a_{t+1}$, which are the conditional expectation of respectively $\alpha_{t}$ and $\alpha_{t+1}$ given obervations $y_{1}, \ldots, y_{t}$, together with their MSEs. When the disturbances are Gaussian white noise, as we have assumed, the conditional expectations are the minimum MSE predictors of the state. Without the Gaussianity assumption, the state estimates are minimum MSE amongst the set of linear predictors.

The Kalman gain $K_{t}$ determines the appropriate weighting of the observations for the computation of the conditional expectations and variances. The smoothed state, that is the expectation of the state vector conditional on the entire sample $y_{1}, \ldots, y_{n}$, can be computed with an additional set of recursions.

If the variables in $\alpha_{t}$ are stationary, the initial mean and covariance matrix are implied by their unconditional distributions. The distributions of non-stationary variables in $\alpha_{t}$ are degenerate and we let the associating diagonal elements in $P_{1}$ approach infinity. We often refer to non-stationary variables in the state vector as diffuse variables, and the Kalman filter requires a diffuse initialisation. Here, we describe the initialisation method by Koopman and Durbin (2003). When the state vector contains diffuse variables, the mean square error matrix $P_{t}$ can be decomposed into a part associated with diffuse state elements $P_{\infty, t}$, and a part where the state has a proper distribution $P_{*, t}$, that is

$$
P_{t}=k P_{\infty, t}+P_{*, t}, \quad k \rightarrow \infty
$$

For the diffuse initial state elements, the corresponding entries on the diagonal matrix $P_{\infty, 1}$ are set to positive values, while the remainder of the matrix contains zeros. Koopman and Durbin show that for models with diffuse state elements the standard Kalman filter can be split into two parts by expanding the inverse of $f_{t}=k f_{\infty, t}+f_{*, t}$ in $k^{-1}$. In the first $d$ iterations of the filter, the state contains diffuse elements, which is indicated by a non-zero 
$P_{\infty, t}$. Separate update equations are maintained for the parts associated with $P_{\infty, t}$ and with $P_{*, t}$. Generally $P_{\infty, t}$ becomes zero after some iterations, after which the standard Kalman filter can be used. The diffuse filter equations for the initial iterations are given by

$$
\begin{aligned}
v_{t} & =y_{t}-Z_{t} a_{t}-c_{t}, & & \\
f_{\infty, t} & =Z_{t} P_{\infty, t} Z_{t}^{\prime}, & f_{*, t} & =Z_{t} P_{*, t} Z_{t}^{\prime}+\sigma_{\varepsilon}^{2}, \\
K_{\infty, t} & =P_{\infty, t} Z_{t}^{\prime} f_{\infty, t}^{-1}, & K_{*, t} & =\left(P_{*, t} Z_{t}^{\prime}-K_{\infty, t} f_{*, t}\right) f_{\infty, t}^{-1}, \\
P_{\infty, t \mid t} & =P_{\infty, t}-f_{\infty, t} K_{\infty, t} K_{\infty, t}^{\prime}, & P_{*, t \mid t} & =P_{*, t}-K_{\infty, t} Z_{t} P_{*, t}^{\prime}-K_{*, t} Z_{t} P_{\infty, t}^{\prime}, \\
P_{\infty, t} & =T_{t} P_{\infty, t \mid t} T_{t}^{\prime}, & P_{*, t} & =T_{t} P_{*, t \mid t} T_{t}^{\prime}+H_{t}, \\
a_{t \mid t} & =a_{t}+K_{\infty, t} v_{t}, & a_{t+1} & =d_{t}+T_{t} a_{t \mid t},
\end{aligned}
$$

when $f_{\infty, t}>0$. In case $f_{\infty, t}$ is zero $K_{\infty, t}$ does not exist, and the equations for $K_{*, t}, P_{\infty, t}, P_{*, t}$ and $a_{t \mid t}$ are given by

$$
\begin{aligned}
K_{*, t} & =P_{*, t} Z_{t}^{\prime} f_{*, t}^{-1}, & a_{t \mid t} & =a_{t}+K_{*, t} v_{t} \\
P_{\infty, t \mid t} & =P_{\infty, t}, & P_{*, t \mid t} & =P_{*, t}-K_{*, t} Z_{t} P_{*, t}^{\prime} .
\end{aligned}
$$

\section{B. Extended Kalman filter with diffuse initialisation.}

A non-linear state space model for a univariate time series $y_{1}, \ldots, y_{n}$ can be defined by the equations

$$
\begin{aligned}
& y_{t}=Z_{t}\left(\alpha_{t}\right)+\varepsilon_{t}, \quad \varepsilon_{t} \sim \operatorname{NID}\left(0, \sigma_{\varepsilon}^{2}\right), \\
& \alpha_{t+1}=T_{t}\left(\alpha_{t}\right)+\eta_{t}, \quad \eta_{t} \sim \operatorname{NID}\left(0, H_{t}\right), \quad t=1, \ldots, n,
\end{aligned}
$$

The observations $y_{t}$ are modeled as a transformation $Z_{t}(\cdot)$ of the latent stochastic state vector $\alpha_{t}$ plus observation noise $\varepsilon_{t}$. The state vector evolves according to the transformation $T_{t}(\cdot)$ and accumulates additional transition noise $\eta_{t}$ at each time $t$. We also assume all the noise terms and the initial state to be mutually independent.

The extended Kalman filter equations provide approximate estimates of the state by applying the standard Kalman filter to the Taylor approximations of (35) expanded around the estimated state from the filter. The first order approximations to the observation and transition equations are given by

$$
\begin{aligned}
y_{t} & \approx Z_{t}\left(a_{t}\right)+\tilde{Z}_{t} \cdot\left(\alpha_{t}-a_{t}\right)+\varepsilon_{t}, \\
\alpha_{t+1} & \approx T_{t}\left(a_{t \mid t}\right)+\tilde{T}_{t} \cdot\left(\alpha_{t}-a_{t \mid t}\right)+\eta_{t},
\end{aligned}
$$

where

$$
\tilde{Z}_{t}=\left.\frac{\partial Z(x)}{\partial x}\right|_{x=a_{t}}, \quad \tilde{T}_{t}=\left.\frac{\partial T(x)}{\partial x}\right|_{x=a_{t \mid t}},
$$

as the predicted and filtered states $a_{t}$ and $a_{t \mid t}$ respectively, are the most recent state estimates available when the the linearizations are required in the filter equations.

As the first order approximation to the model is linear in $\alpha_{t}$, we can apply the Kalman filter of (31) to (36), where the non-random terms in the linearized model $Z_{t}\left(a_{t}\right)-\tilde{Z}_{t} a_{t}$ and $T_{t}\left(a_{t \mid t}\right)-\tilde{T}_{t} a_{t \mid t}$ are incorporated into $c_{t}$ and $d_{t}$ respectively. This yields the equations

$$
\begin{aligned}
v_{t} & =y_{t}-Z_{t}\left(a_{t}\right), & f_{t} & =\tilde{Z}_{t} P_{t} \tilde{Z}_{t}^{\prime}+\sigma_{\varepsilon}^{2}, \quad K_{t}=P_{t} \tilde{Z}_{t}^{\prime} f_{t}^{-1}, \\
a_{t \mid t} & =a_{t}+K_{t} v_{t}, & P_{t \mid t} & =P_{t}-f_{t} K_{t} K_{t}^{\prime}, \\
a_{t+1} & =T_{t}\left(a_{t \mid t}\right), & P_{t+1} & =\tilde{T}_{t} P_{t \mid t} \tilde{T}_{t}^{\prime}+H_{t},
\end{aligned}
$$


which comprise the extended Kalman filter for the non-linear state space model (35). A more detailed exposition of the extended Kalman filter can be found in Jazwinski (1970) or Anderson and Moore (1979).

The filter equations provides estimates of the predicted and filtered state $a_{t+1}$ and $a_{t \mid t}$ with approximate mean square error matrices $P_{t+1}$ and $P_{t \mid t}$. The smoothed states $\hat{\alpha}_{t}$, which are conditioned on the entire sample of observations, can be calculated with an additional set of recursions running from $t=n$ backwards to $t=1$, see Durbin and Koopman (2001). For the first order approximation to the non-linear model, these take the form of

$$
L_{t}=\tilde{T}_{t}-\tilde{T}_{t} K_{t} \tilde{Z}_{t}, \quad r_{t-1}=\tilde{Z}_{t}^{\prime} f_{t}^{-1} v_{t}+L_{t}^{\prime} r_{t}, \quad \hat{\alpha}_{t}=a_{t}+P_{t} r_{t-1}
$$

which is initialised by $r_{n}=0$.

When we apply the diffuse filter to the linear approximation (36), we obtain the diffuse extended Kalman filter for $t=1, \ldots, d$, given by

$$
\begin{aligned}
v_{t} & =y_{t}-Z_{t}\left(a_{t}\right), & & \\
f_{\infty, t} & =\tilde{Z}_{t} P_{\infty, t} \tilde{Z}_{t}^{\prime}, & f_{*, t} & =\tilde{Z}_{t} P_{*, t} \tilde{Z}_{t}^{\prime}+\sigma_{\varepsilon}^{2}, \\
K_{\infty, t} & =P_{\infty, t} \tilde{Z}_{t}^{\prime} f_{\infty, t}^{-1}, & K_{*, t} & =\left(P_{*, t} \tilde{Z}_{t}^{\prime}-K_{\infty, t} f_{*, t}\right) f_{\infty, t}^{-1}, \tilde{Z}_{\infty, t} \tilde{Z}_{t} P_{*, t}^{\prime}-K_{*, t} P_{\infty, t}^{\prime}, \\
P_{\infty, t \mid t} & =P_{\infty, t}-f_{\infty, t} K_{\infty, t} K_{\infty, t}^{\prime}, & P_{*, t \mid t} & =P_{*, t}-K_{\infty, t} \tilde{Z}_{t} P_{t} \tilde{T}_{*, t} \tilde{T}_{t}^{\prime}+H_{t}, \\
P_{\infty, t} & =\tilde{T}_{t} P_{\infty, t \mid t} \tilde{T}_{t}^{\prime}, & P_{*, t} & =\tilde{T}_{t} P_{*+1} \\
a_{t \mid t} & =a_{t}+K_{\infty, t} v_{t}, & a_{t+1} & =T_{t}\left(a_{t \mid t}\right),
\end{aligned}
$$

for $f_{\infty, t}>0$, and

$$
\begin{aligned}
K_{*, t} & =P_{*, t} \tilde{Z}_{t}^{\prime} f_{*, t}^{-1}, & a_{t \mid t} & =a_{t}+K_{*, t} v_{t} \\
P_{\infty, t \mid t} & =P_{\infty, t}, & P_{*, t \mid t} & =P_{*, t}-K_{*, t} \tilde{Z}_{t} P_{*, t}^{\prime}
\end{aligned}
$$

for $f_{\infty, t}=0$. When $P_{\infty, t}$ becomes zero after $d$ iterations, the standard EKF of (38) applies with $P_{d}=P_{*, d}$.

The state smoothing equations (39) can be split in a similar manner. The diffuse extended smoothing equations when $f_{\infty, t}>0$ are

$$
\begin{aligned}
L_{\infty, t} & =\tilde{T}_{t}-\tilde{T}_{t} K_{\infty, t} \tilde{Z}_{t} \\
r_{t-1}^{(0)} & =L_{\infty, t}^{\prime} r_{t}^{(0)} \\
r_{t-1}^{(1)} & =\tilde{Z}_{t}^{\prime}\left(f_{\infty, t}^{-1} v_{t}-K_{*, t}^{\prime} r_{t}^{(0)}\right)+L_{\infty, t}^{\prime} r_{t}^{(1)}
\end{aligned}
$$

while for $f_{\infty, t}=0$ we have

$$
\begin{aligned}
L_{*, t} & =\tilde{T}_{t}-\tilde{T}_{t} K_{*, t} \tilde{Z}_{t}, \\
r_{t-1}^{(0)} & =\tilde{Z}_{t}^{\prime} f_{*, t}^{-1} v_{t}+L_{*, t}^{\prime} r_{t}^{(0)}, \\
r_{t-1}^{(1)} & =\tilde{T}_{t}^{\prime} r_{t}^{(1)},
\end{aligned}
$$

running from $t=d, \ldots, 1$ with $r_{d}^{(0)}=r_{d}$ and $r_{d}^{(1)}=0$. The smoothed state is calculated as

$$
\hat{\alpha}_{t}=a_{t}+P_{*, t} r_{t-1}^{(0)}+P_{\infty, t} r_{t-1}^{(1)} .
$$


We usually display the smoothed estimates of the state vector once the unknown parameters are estimated. In models without diffuse variables, the parameter vector $\theta$ can be estimated by maximising the Gaussian log-likelihood function as given by

$$
\log L(\theta)=-\frac{1}{2} \sum_{t=1}^{n} \log \left(2 \pi f_{t}\right)-\frac{1}{2} \sum_{t=1}^{n} v_{t}^{2} / f_{t}
$$

where the one-step prediction error $v_{t}$ and its variance $f_{t}$ are obtained from the linear or extended Kalman filter. The diffuse likelihood function is given by

$$
\log L=-\frac{n}{2} \log 2 \pi-\frac{1}{2} \sum_{t=1}^{d} w_{t}-\frac{1}{2} \sum_{t=d+1}^{n}\left(\log f_{t}+f_{t}^{-1} v_{t}^{2}\right)
$$

where $w_{t}=\log f_{\infty, t}$ for $f_{\infty, t}>0$ or $w_{t}=\log f_{\infty, t}+f_{*, t}^{-1} v_{t}^{2}$ when $f_{\infty, t}=0$. 


\section{References}

Anderson, B. D. O. and J. B. Moore (1979). Optimal Filtering. Englewood Cliffs: PrenticeHall.

Bowerman, B. L., A. B. Koehler, and D. J. Pack (1990). Forecasting time series with increasing seasonal variation. Journal of Forecasting 9, 419-436.

Carter, C. K. and R. Kohn (1994). On Gibbs sampling for state space models. Biometrika 81, 541-53.

Cecchetti, S. G., A. K. Kashyap, and D. W. Wilcox (1997). Interactions between the seasonal and business cycles in production and inventories. The American Economic Review 8\%, 884-892.

Commandeur, J. J. F. and S. J. Koopman (2007). An Introduction to State Space Time Series Analysis. Oxford: Oxford University Press.

de Freitas, N., A. Doucet, and N. Gordon (2001). Sequential Monte Carlo Methods in Practice. New York: Springer Verlag.

Doornik, J. A. (2007). Object-Oriented Matrix Programming using Ox 5.0. London: Timberlake Consultants Ltd. See http://www.doornik.com.

Durbin, J. and S. J. Koopman (2000). Time series analysis of non-Gaussian observations based on state space models from both classical and Bayesian perspectives (with discussion). J. Royal Statistical Society B 62, 3-56.

Durbin, J. and S. J. Koopman (2001). Time Series Analysis by State Space Methods. Oxford: Oxford University Press.

Durbin, J. and M. Murphy (1975). Seasonal adjustment based on a mixed additive - multiplicative model. J. Royal Statistical Society A 138, 385-410.

Findley, D., B. Monsell, W. Bell, M. Otto, and B.-C. Chen (1998). New capabilities of the $\mathrm{X}$-12-ARIMA seasonal adjustment program (with discussion). J. Business and Economic Statist. 16, 127-77.

Franses, P. H. (1995). Quarterly us unemployment: cycles, seasons and asymmetries. Empirical Economics 20,717-725.

Franses, P. H. and P. T. de Bruin (1999). Seasonal adjustment and the business cycle in unemployment. Econometric Institute Report 152, Erasmus University Rotterdam, Econometric Institute.

Fruhwirth-Schnatter, S. (1994). Bayesian model discrimination and Bayes factors for state space models. J. Royal Statistical Society B 56, 237-46.

Gersch, W. and G. Kitagawa (1983). The prediction of time series with trends and seasonalities. J. Business and Economic Statist. 1, 253-64.

Gordon, N. J., D. J. Salmond, and A. F. M. Smith (1993). A novel approach to non-linear and non-Gaussian Bayesian state estimation. IEE-Proceedings F 140, 107-13. 
Harvey, A. C. (1989). Forecasting, Structural Time Series Models and the Kalman Filter. Cambridge: Cambridge University Press.

Harvey, A. C. and A. Scott (1994). Seasonality in dynamic regression models. Economic Journal 104, 1324-45.

Jazwinski, A. H. (1970). Stochastic Processes and Filtering Theory. New York: Academic Press.

Koopman, S. J. and J. Durbin (2003). Filtering and smoothing of state vector for diffuse state space models. J. Time Series Analysis 24, 85-98.

Koopman, S. J., N. Shephard, and J. A. Doornik (1999). Statistical algorithms for models in state space form using SsfPack 2.2. Econometrics Journal 2, 113-66. http://www.ssfpack.com/.

Osborn, D. R. and A. Matas-Mir (2004). Does seasonality change over the business cycle? an investigation using monthly industrial production series. European Economic Review 48 , 1309-1332.

Ozaki, T. and P. Thomson (1994). A non-linear dynamic model for multiplicative seasonaltrend decomposition. Journal of Forecasting 21, 107-124.

Pitt, M. K. and N. Shephard (1999). Filtering via simulation: auxiliary particle filter. J. American Statistical Association 94, 590-9.

Proietti, T. (2000). Comparing seasonal components for structural time series models. International Journal of Forecasting 16, 247-260.

Proietti, T. and M. Riani (2006). Transformations and seasonal adjustment. In Eurostat conference on seasonality, seasonal adjustment and their implications for short-term analysis and forecasting.

Shephard, N. (1994). Partial non-Gaussian state space. Biometrika 81, 115-31.

Shephard, N. and M. K. Pitt (1997). Likelihood analysis of non-Gaussian measurement time series. Biometrika 84, 653-67.

van Dijk, D., B. Strikholm, and T. Teräsvirta (2003). The effects of institutional and technological change and business cycle fluctuations on seasonal patterns in quarterly industrial production series. Econometrics Journal 6, 79-98. 